\title{
Evaporation-induced evolution of the capillary force between two grains
}

\author{
Boleslaw Mielniczuk • Tomasz Hueckel • \\ Moulay Saïd El Youssoufi
}

Received: 15 January 2013

(C) Springer-Verlag Berlin Heidelberg 2014

\begin{abstract}
The evolution of capillary forces during evaporation and the corresponding changes in the geometrical characteristics of liquid (water) bridges between two glass spheres with constant separation are examined experimentally. For comparison, the liquid bridges were also tested for mechanical extension (at constant volume). The obtained results reveal substantial differences between the evolution of capillary force due to evaporation and the evolution due to extension of the liquid bridges. During both evaporation and extension, the change of interparticle capillary forces consists in a force decrease to zero either gradually or via rupture of the bridge. At small separations between the grains (short \& wide bridges) during evaporation and at large volumes during extension, there is a slight initial increase of force. During evaporation, the capillary force decreases slowly at the beginning of the process and quickly at the end of the process; during extension, the capillary force decreases quickly at the beginning and slowly at the end of the process. Rupture during evaporation of the bridges occurs most abruptly
\end{abstract}

\section{B. Mielniczuk}

Institut de Radioprotection et de Sûreté Nucléaire, IRSN,

B.P. 3, 13115 Saint-Paul-lez-Durance Cedex, France

B. Mielniczuk · M. S. El Youssoufi

Laboratoire de Micromécanique et d'Intégrité des Structures

MIST, IRSN-CNRS-Université Montpellier 2,

Montpellier, France

e-mail: boleslaw.mielniczuk@univ-montp2.fr

T. Hueckel ( $\varangle)$

Duke University, Durham, NC 27708, USA

e-mail: hueckel@duke.edu

M. S. El Youssoufi

Laboratoire de Mécanique et Génie Civil UMR UM2-CNRS 5508, Université Montpellier 2, 34095 Montpellier Cedex 5, France

e-mail: Moulay-Said.El-Youssoufi@univ-montp2.fr for bridges with wider separations (tall and thin), sometimes occurring after only $25 \%$ of the water volume was evaporated. The evolution (pinning/depinning) of two geometrical characteristics of the bridge, the diameter of the threephase contact line and the "apparent" contact angle at the solid/liquid/gas interface, seem to control the capillary force evolution. The findings are of relevance to the mechanics of unsaturated granular media in the final phase of drying.

Keywords Capillary force - Capillary bridge . Evaporation $\cdot$ Contact angle $\cdot$ Surface tension

\section{Introduction and background}

The assessment of the interparticle forces that arise in capillary liquid bridges and their evolution, including their demise, are of primary importance because of their contribution to the formation, deformation and flow of particulate materials, including soils, and in numerous technologies, including immersion lithography, fiber coating, inkjet printing, photonic crystal production, etc. The capillary forces between partially wet grains are thought to impart an apparent macroscopic or "sand-castle" strength to the moist granular materials, even in the absence of the intrinsic cohesion or confining stress (see e.g. [1-7]). Two-grain systems are important not only because they are the most elementary but also because more complex multi-grain liquid bridges will eventually break down to a number of two-grain systems (see e.g. [8-10]).

The objective of the presented experiments is to better understand the evaporation-induced evolution of capillary forces in a two-grain liquid bridge at different but constant separations. To our knowledge, no comprehensive studies on capillary bridge evaporation have been undertaken. There are 
a few notable exceptions of a specifically focused work on nano-scale separations $(<100 \mathrm{~nm})$ by Gao [11] and Maeda et al. [12], or spheres at direct contact, Cutts and Burns [13], or on drying of colloidal crystals by Zhou et al. [14]. Most of the previous studies on the capillary forces in two-grain liquid bridges deal with extension of the bridges at a constant liquid volume or a constant suction [12,15-18].

Some of the fundamental questions related to the evolution of the evaporating liquid bridges may have been answered through studies of sessile drop evaporation. The primary such questions are related to the contact angle $(C A)$ hysteresis, the contact diameter $(C D)$ pinning and depinning, and how these properties affect the evaporation and the capillary forces. We shall explore to some degree the analogies between drying sessile drops and liquid bridges, based on the vast amount of recent work on the former subject (e.g. [19-24]). However, a central point here is the evolution of the capillary force.

To place the obtained results on evaporation in a wider context, a parallel series of extension tests is performed on the capillary bridges. In these tests, the grain separation is increased from zero at a constant rate at constant liquid volumes of different amounts.

The shape of the capillary bridge at equilibrium is represented by a set of variables used to determine the capillary pressure in the Laplace-Young equation (see e.g. $[25,26]$ ). These variables include two quantities representing the two principal surface curvatures of the bridge gorge measured at its neck and the volume of the liquid. The form of the surface of the phase interface is usually determined through minimization of the total free energy of the bridge, under a condition of constant volume [26]. Numerous efforts were undertaken to determine the shape of the interface $[15-18,27,28]$. The surface curvatures determine also the single quantity of the pressure difference across the liquid-gas interface (Laplace pressure) and its resultant across the bridge gorge area as well as the surface tension force acting at the bridge perimeter. The two forces result in the intergranular capillary force (positive when attractive). Additionally, from the equilibrium of surface tensions at the solid/liquid/gas interface one can calculate a unique equilibrium contact angle [26].

As mentioned above, in contrast to evaporating capillary bridges, the case of an evaporating sessile droplet has been intensely studied $[19,23,29]$. The key issue that has emerged from these studies is the importance of what happens at the contact of the liquid body with the "real solid surfaces" [20$24,30,31]$. Such surfaces are understood as endowed with surface roughness, which can be enhanced by the presence of precipitated liquid impurities (the coffee ring effect discussed in e.g. Deegan et al. [32]).

The main conclusion from the sessile drop studies concerns the equilibrium contact angle, which is found to be "a principally unmeasurable physical value" [31,33,34], whereas the observable contact angles are either blocked or vary according to the pinning or depinning condition of the "triple" line at the interface of the three phases $[19,21,30,34,35]$.

Hence in our experiments, several other quantities that do not enter the Laplace-Young equation, but are seen to contribute to the evolution of the capillary bridge shape, have also been measured. These are the contact diameters and observable contact angles.

It is realized that the evolution of the shape of the evaporating capillary bridge, and that of the capillary forces is driven by the water mass removal due to evaporation. The evaporation rate is determined by external conditions, namely the external vapor pressure (or relative humidity) and the temperature of the environment, which is kept constant. The aforementioned shape changes may involve the internal viscous flow of liquid, where the internal velocity depends on the evaporation flux. The value of capillary number, $C a(C a=\mu v / \gamma$, where $\mu v$ and $\gamma$ are respectively, viscosity, velocity and surface tension of the liquid) of $10^{-4}$ is considered as a threshold, below which the viscous flow effects are insignificant compared to surface tension effects [36]. In our case, based on the measured value of surface tension of the bridge liquid, and the highest measured value of the velocity of the radial flow (see a separate paper [37]), the capillary number was found to be about $4 \times 10^{-7}$ for 0.7 and $2 \mathrm{~mm}$ of separation. As a result, the liquid bridge departs due to evaporation from the initial state of mechanical equilibrium, but for a relatively low evaporation rate the latter can be approximated by a series of quasi-stable states that are not far from equilibrium, each state having incrementally different geometries [36].

In reference [37], we report the calculated Laplace pressure and surface tension forces as they evolve during evaporation, whereas results concerning the rupture of the bridges are presented in [9].

\section{Experimental conditions and techniques}

The investigated system consists of two precision glass spheres (grade 25) and a capillary bridge between them formed by an instilled liquid. The spheres are $8 \mathrm{~mm}$ in diameter. The evaporation of the capillary bridge is conducted at a constant relative humidity of $25 \%$ in a climate-controlled chamber at a constant temperature of $21^{\circ} \mathrm{C}$. Corresponding experiments with the liquid bridge extension are performed using the same systems.

The vertical configuration of the spheres has been chosen for most of the experiments as the capillary force measurement relies on gravity. All calculations are performed for such an arrangement. Because the water volumes are so 
Fig. 1 Apparatus configuration

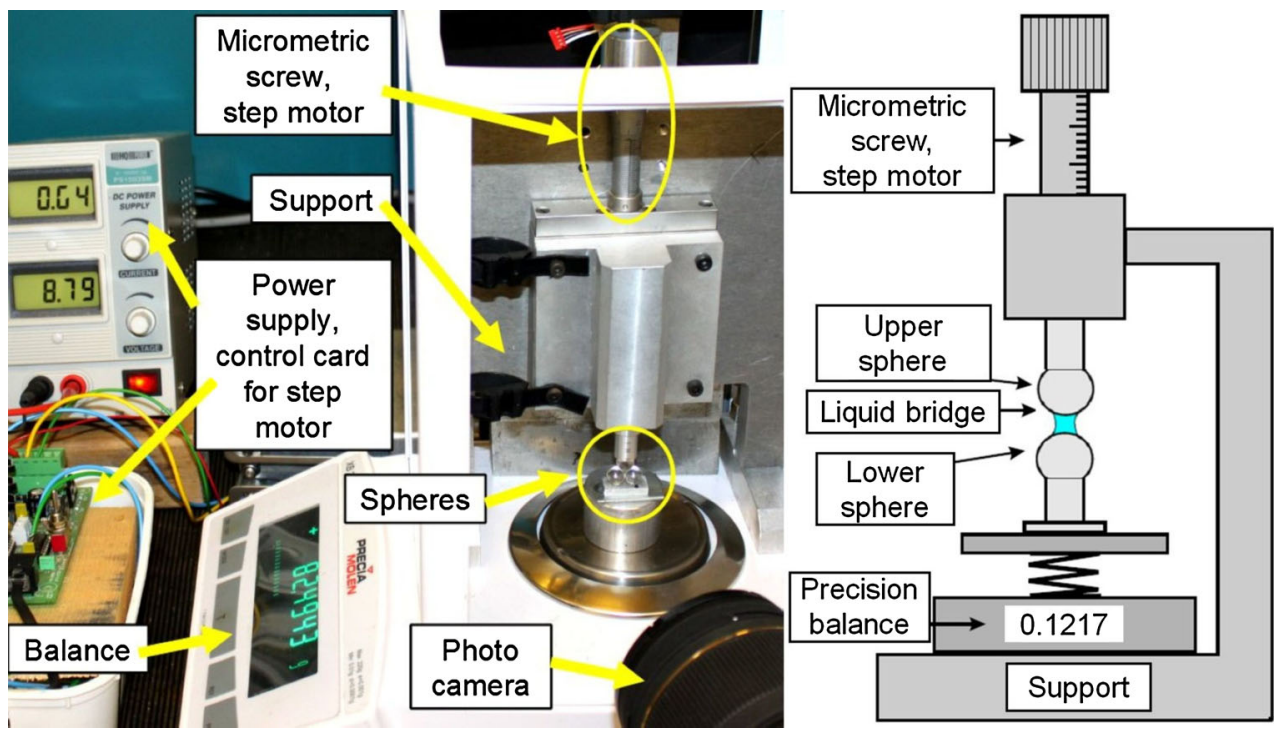

small, gravity is negligible compared to the other forces, and the vertical and horizontal configurations can be as assumed to be equivalent (see e.g. [15]).

The bottom sphere is fixed to the substrate and is placed on a balance Precia Molen XB220A, with precision of $10^{-4}$ gram. The top sphere is attached with epoxy glue Epolam 2010 (creep effects of the glue are negligible) to the micrometer table and is suspended from the support without contact with the balance. The centers of the spheres are collinear with the axis of vertical symmetry of the balance. The setup is derived from an earlier device described in detail by Soulié et al. [38] and Gras et al. [18].

The separation between the spheres for the evaporation experiments is adjusted by a micrometer screw (see Fig. 1) with accuracy of $0.01 \mathrm{~mm}$. Sphere separation can also be changed smoothly with a constant speed by a step motor for the extension tests.

The liquid is a mixture being used at the University of Montpellier-2 for experiments on capillary forces, see e.g. Gras et al. [18]. The mixture composes of the milli-Q ultrapure water and some ingredients acquired by water when it is circulated through the hydraulic system of the apparatus (not relevant in our experiments). The liquid has been frequently tested for surface tension $\gamma_{s}$ using the Wilhelmy plate method (see, e.g., [25]). The mean value from 17 tests of the liquids freshly harvested from the liquid bridge and those exposed to the atmosphere for over $1.5 \mathrm{~h}$, equivalent to the duration of the evaporation test, was determined as $\gamma_{s}=0.0496 \mathrm{~N} / \mathrm{m}$, with a standard deviation of $0.0029 \mathrm{~N} / \mathrm{m}$ $(5.7 \%)$. A change in surface tension of individual liquid samples over the period of 1.5 hour is typically less than the $3 \%$. Similar values were reported by Gras (mean value of $\gamma_{s}=0.051 \mathrm{~N} / \mathrm{m}$ [18]). These values are notably lower than the nominal value of $0.0728 \mathrm{~N} / \mathrm{m}$ for the surface tension of distilled water. Surface tension is notoriously sensitive to the presence of contaminants (see, e.g. [39,40]). All the force related results are any way normalized with respect to surface tension.

Before each experiment, the spheres are washed with $95 \%$ ethanol and dried. A procedure for the placement of water and the formation of the bridge is standardized as it impacts the initial contact angles (see $[15,41,42]$ ). The procedure includes: (i) setting the separation between the spheres; (ii) injecting a specified volume of water onto the lower tip of the top sphere with a laboratory micro-syringe of $0.1 \mu \mathrm{l}$ accuracy; and (iii) allowing the injected water to drip into the gap between the spheres. The droplet attached under the upper sphere initial grows in height, and upon reaching the lower sphere, it transforms into the capillary bridge. For the extension tests, the initial separation is zero and water immediately in contact with both spheres, forms a ring-like initial capillary bridge.

The evolution of the capillary bridge during evaporation presented here is for a single initial liquid volume of $4 \mu \mathrm{l}$ at constant separations ranging from $D=0.01$ to $2.0 \mathrm{~mm}$. In terms of non-dimensional separations defined as $\xi=D / 2 R$ (see Fig. 2a), these relative separations are $\xi=0.00125$ to 0.250 . At the instant the liquid is added, the reading of the balance carrying the lower sphere decreases as a result of the development of the attractive force in the liquid bond between the spheres. This force, $F_{\text {int }}$, referred to as the total intergranular force, is calculated as $F_{i n t}=\left(\mathrm{m}_{d r y}-m_{b a l}\right) g$, where $m_{d r y}$ is the mass of the bottom sphere when dry, $m_{b a l}$ is the reading of the balance supporting the bottom sphere and the liquid bridge during drying, and $g$ is the acceleration due to gravity.

The quantity of $4 \mu 1$ of water was experimentally selected as an optimal amount both to repeatedly produce a symmetric 

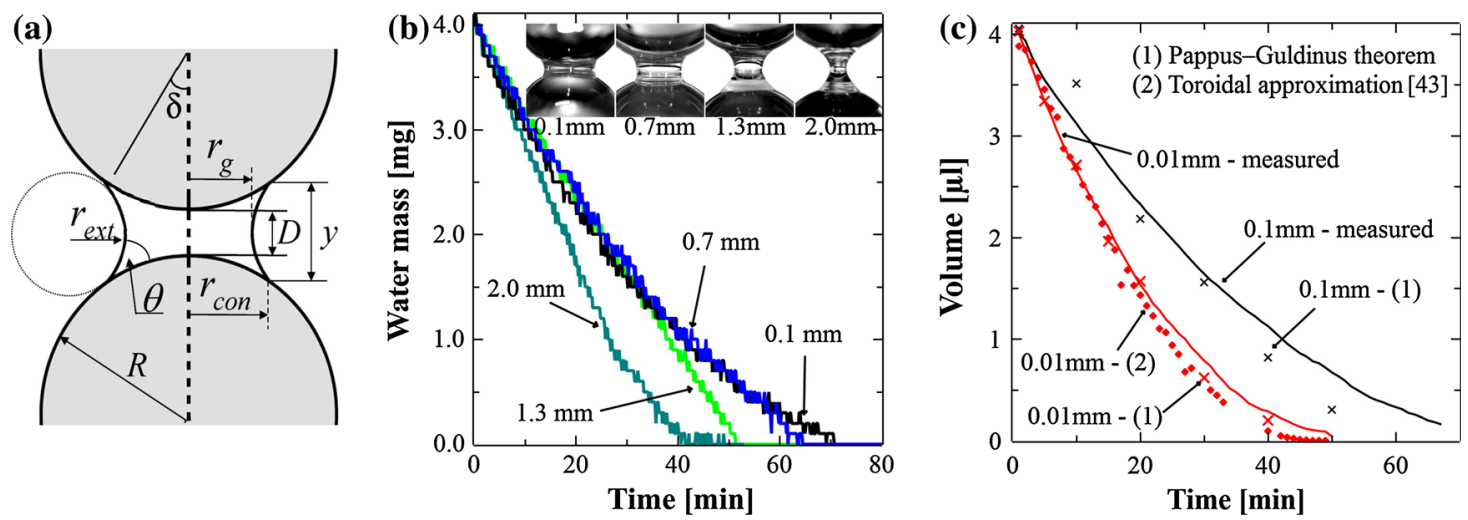

Fig. 2 (a) Schematic view of the capillary bridge between two identical spheres; (b) Evolution of the mass of a capillary bridge between two particles due to evaporation. Inset: initial shape of the bridge at separa-

capillary bridge at all analyzed separations and to minimize the influence of gravity on the bridge shape.

For the extension tests, the apparatus configuration, preparation procedure and data processing methods are the same as those for the evaporation tests. The spheres are initially placed in contact with one another, after which the liquid bridge with a pre-determined volume from 0.1 to $10 \mu 1$ is formed. The top sphere is then lifted with the use of a step motor at a constant speed of $16 \mu \mathrm{m} / \mathrm{s}$ while the capillary force is continuously measured. The extension rate is chosen both to minimize the evaporation during the test (the duration is about $2-3 \mathrm{~min}$ ) and to minimize the influence of water viscosity.

A still camera Canon EOS500 with a macro lens is used to image the evolution of the bridge geometry during both processes. Images are saved each minute for the evaporation tests and each $5 \mathrm{~s}$ for the extension tests, with a resolution of 12 megapixels. The images are processed by Fiji (Image J) software. The bridge characteristics are determined from the images using MBRuler Pro and a Matlab program.

The capillary force data were acquired every $10 \mathrm{~s}$ for the evaporation tests and every $1 \mathrm{~s}$ for the extension tests. Each of the tests has been repeated several times, and the results display some scatter (see, e.g., Fig. 4) presumably due to a non-symmetric initial shape of the bridge (errors up to $10 \%$ ). Bridges that did not display rotational symmetry or symmetry across the horizontal mid-plane were rejected. The reported results are mean values, with the outliers excluded from the mean calculations.

\section{Experimental results}

The calibrated balance records and image processing allowed us to retrieve a number of key physical and geometric characteristics of the capillary bridge as functions of time (see Fig. 2a). These characteristics are listed as follows: (i) liq- tion distances of $0.1,0.7,1.3$ and $2.0 \mathrm{~mm}$. The initial liquid volume is $4 \mu \mathrm{l}$; (c) Validation of the water body volume determined in the parallel test (see text)

uid water mass loss; (ii) resultant capillary force $F_{C A P}$; (iii) radius of curvature of the surface of the water body measured at the equator, $r_{g}$ (called the gorge radius or "neck" radius); (iv) radius of the external curvature of the meniscus along a meridian of the bridge surface (assuming rotational symmetry) $r_{\text {ext }}$; (v) length of the external bridge profile $l$ (slant height, considering frustum curvature) and hence the interface surface area (assuming rotational symmetry); (vi) apparent contact angle $\theta$; (vii) half-filling angle $\delta$ and (viii) contact radius, $r_{c o n}$. The contact radius refers to a "triple" line of contact of gas/solid/liquid phases. It is assumed that the triple line is a circle, and the radius reported is an average of the four "radii" of the bridge visible in the images. The apparent contact angle is an angle extrapolated from a point on the interface surface vertical intersection at a distance of $5 \mu \mathrm{m}$ from the triple line, using MBRuler Pro software. The apparent contact angle is different from the equilibrium contact angle.

The above measurements allow us to calculate the following variables: (1) relative volume loss; (2) evaporating surface area; (3) vapor flux evolution during evaporation (from the evaporation surface); and (4) total capillary force. The calculations of the suction and surface tension components of the capillary force and the subsequent analysis are presented in a separate paper [37].

\subsection{Mass loss of liquid water}

The variable driving the evolution process of the liquid bridge during drying is the rate of the loss of evaporating liquid mass (vapor flux, when referred to the unit interface area), which is measurable but not controllable. The mass flux results from the difference between the constant ambient vapor pressure in the testing chamber and the saturated vapor pressure. In the following analyses, this difference is considered to be the independent variable that affects the other variables, such 
as the bridge volume, bridge profile shape, suction, wetting angle, capillary force, etc. The evaporated mass is measured in a duplicate experiment, in which the entire water bridge and both spheres at the same separation sit directly on the balance, which therefore measures only the decreasing mass of the liquid bridge water. From the point of geometry of the liquid body and its force relationship with the solid spheres both experiments are identical. These measurements were validated by comparing to the volume of the water body approximated as axially symmetric from the area of the projection of the bridge in the images using Pappus-Guldinus theorem for selected separations (Fig. 2c) and to a volume calculated employing a toroidal approximation using current geometric data from image processing (as discussed later) following the formula developed in [43]. The difference between the experiments shown is less than a standard deviation in the measurements. It tends to be higher as the process advances, but still below the experimental error. The mass evolution for an initial bridge volume of $4 \mu 1$ is shown for different separations in Fig. 2b.

Evaporation curves exhibit initially a linear relationship between the total mass and time (i.e., a constant evaporation rate), and in a later stage a decreasing evaporation rate. The slope of the linear part of the curve varies with the separation distance; i.e.: $0.112 \mathrm{mg} / \mathrm{min}$ at $2.0 \mathrm{~mm}, 0.078 \mathrm{mg} / \mathrm{min}$ at $1.3 \mathrm{~mm}, 0.073 \mathrm{mg} / \mathrm{min}$ at $0.7 \mathrm{~mm}$, and $0.072 \mathrm{mg} / \mathrm{min}$ at $0.1 \mathrm{~mm}$. For separations that range from 0.1 to $0.7 \mathrm{~mm}$, the liquid mass evolution is approximately the same. For wider separations with the same initial volume, the evaporation rates are higher and duration of the whole process is much shorter.

\subsection{Evaporation surface area and evaporation flux}

For the evaporation experiments, it is necessary to evaluate the surface of evaporation area, $S$, which is an important geometrical characteristics of the liquid bridge, as it directly affects the calculated evaporation flux, Fig. 3 a.
The surface area of the interface has been obtained from the image processing of the projections of the evolving bridge contour. In all cases, the surface area of evaporation decreases almost linearly with the diminishing volume of the liquid bridge. At lower separations, the evolutions of the surface areas are similar to each other, but for higher separations the area is significantly larger than that for lower separations. It is also interesting to note that the evolution of the average evaporation flux, calculated as the ratio between the total evaporating mass per second and the evaporating surface area, is roughly the same for all separations, as seen in Fig. 3b. The average evaporation flux is nearly constant for the first half of the process, after which the evaporation accelerates substantially, especially for small separations.

\subsection{Capillary force evolution}

Capillary force, $F_{C A P}$, is obtained from total intergranular force, $F_{\text {int }}$, measured using the precision balance. The capillary force is calculated from the total intergranular force by subtracting the force due to the hydrostatic pressure, which is very small, at the neck, following Princen [28] and Adams et al. [44]. The buoyancy contribution is also found to be negligible (see [15]).

The value of the initial capillary force, which is the force at time zero, is equivalent to the capillary force at thermodynamic equilibrium because no evaporation influence could have taken effect. The initial value depends strongly on the grain separation, or, in more "structural" terms, on the slenderness of the bridge. In general, the initial capillary attraction is higher for more narrow separations (i.e., for flatter bridges), with the exception of the narrowest separations, 0.05 and $0.01 \mathrm{~mm}$ (Fig. 4a, b). This is intuitive when one considers the space between the spheres as a (horizontal) capillary slot; thus, for a narrower slot, there is the higher force. Notably, the smallest separations, which are on the order of tens of micrometers that prove an exception to the rule are not small enough for adhesion or adsorption or van der Waals, forces to play a role [45]. The maximum values
Fig. 3 Evolution of the evaporation surface area (a) and flux (b) during the evaporation of a capillary bridge, for different grain separations. The initial liquid volume is $4 \mu 1$
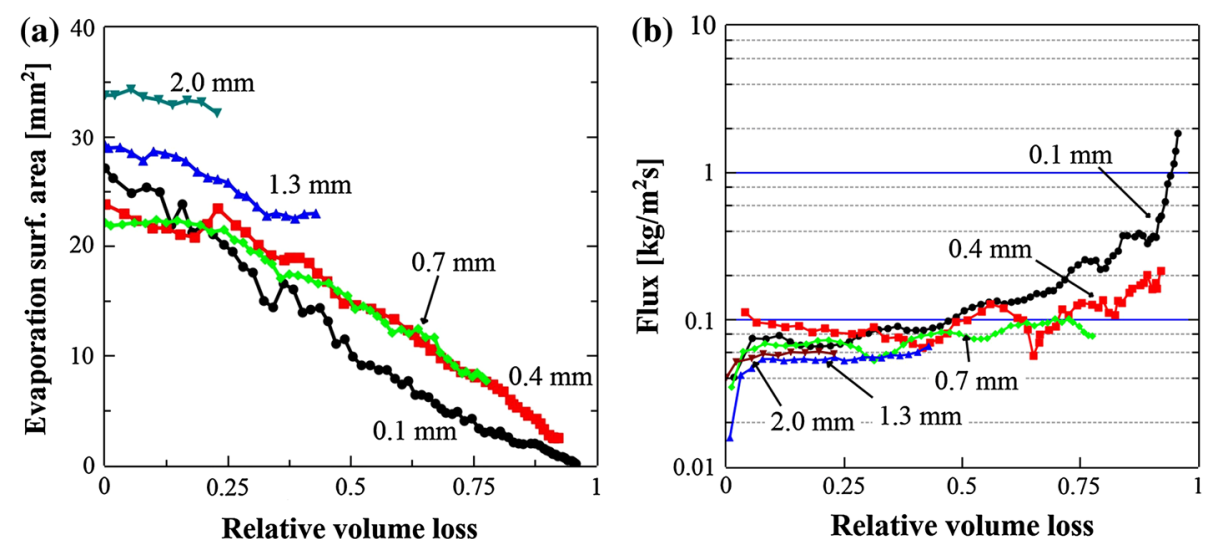

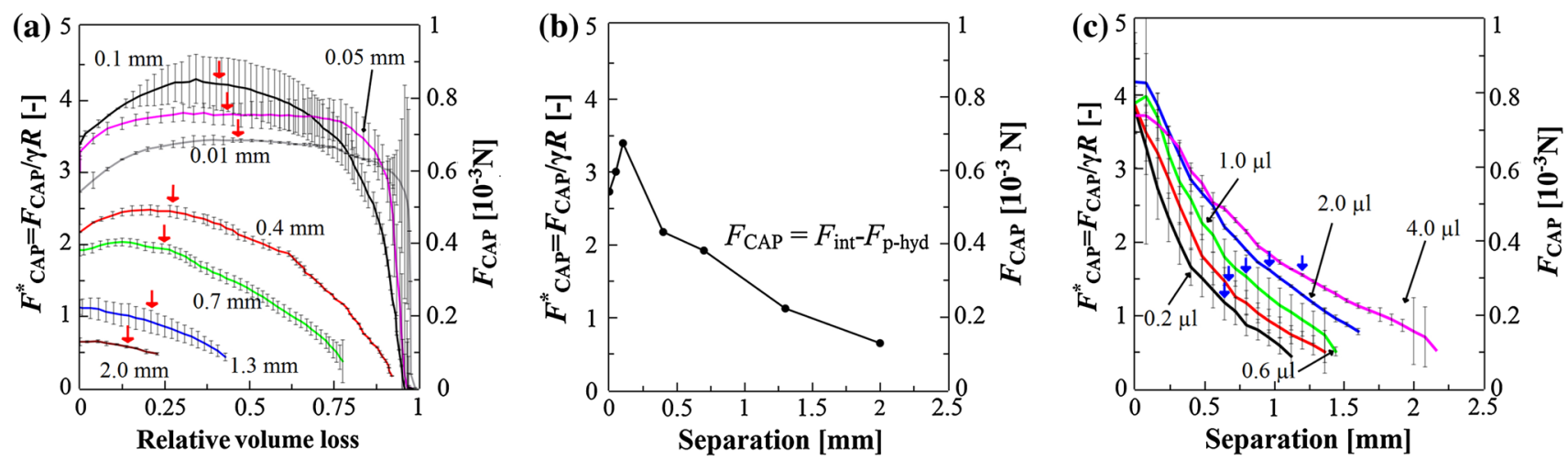

Fig. 4 Evolution of the capillary force between two spheres: (a) during evaporation at different constant separations versus relative volume loss, with an initial liquid volume of $4 \mu$ l. (b) initial force immediately after liquid bridge formation for different separations, liquid volume

of $4 \mu 1$; (c) during an extension test at constant (nominally) different water volumes versus variable separation. The thick arrows in (a) and (c) mark the onset of de-pinning of the triple line (or the onset of change of the "apparent" contact angle variable), as determined from Fig. 9

of the initial force are in the order of $0.7 \times 10^{-3} \mathrm{~N}$, for the flatter bridges, about of $1 \times 10^{-4} \mathrm{~N}$ for the tallest bridge. In terms of the non-dimensionalized values these correspond to 3.6 and 0.6. The former value (for the flattest bridge) calculated by minimizing the interface surface area by Lian et al. [27], using a toroidal approximation of the bridge shape and under the assumption of zero contact angle is of the order of $4-5$, thus about $10-20 \%$ higher. The minimization hypotheses lead in general to overestimations of the experimental values [17]. Hence, our values are clearly of the order of commonly predicted values. A more detailed analysis is provided in "Discussion".

Evolution of the capillary force induced by evaporation (Fig. 4a) exhibits a general trend of an eventual decline of the force, as shown for at a series of constant separations. However, the process occurs in somewhat different modes for three classes of separations: wide, narrow and extremely narrow. These classes can also be described structurally (tall and slender, short and wide, and very short and very wide bridges, respectively), see Fig. $2 b$ inset for proportions, and in terms of their relative separations $(0.250>\xi>0.0875,0.0875>$ $\xi>0.0125$ and $0.0125>\xi>0.00125$, respectively).

At the widest separations, 2 and $1.3 \mathrm{~mm}$, the capillary force decreases monotonically with a modest rate, but abruptly goes to zero as the bridge ruptures at a relatively early stage of the water removal; for these two separations, more than 75 and $50 \%$ respectively, of the capillary water remains in the system after rupture. The value of the force at rupture is a fraction (77 and $34 \%$ respectively) of the initial value.

For all more narrow separations (below $0.7 \mathrm{~mm}$ ), the reduction of the capillary force is preceded by an initial, insignificant increase, which reaches a maximum at the midrange of the volume loss (later for smaller separations). The capillary force value then gradually decreases to zero and accelerates before rupture, which coincides with a complete removal of water.

At the narrowest separations $(0.01$ and $0.05 \mathrm{~mm})$, i.e. for flat bridges, the initial part of the evaporation process features a slight increase of the capillary force, with an extended plateau over a substantial part of the evaporation (between $1 / 4$ and $3 / 4$ of the relative volume loss). The capillary force then decreases continuously, but accelerates when approaching zero in an unstable fashion (i.e. large force changes per incremental change in volume). This stage begins when less than $1 / 10$ of the initial water volume remains. Zero capillary force is reached at a point when there is no more water left.

In general, narrow separations produce higher capillary forces throughout most of the process, with important exceptions for when the separations are less than or equal to $0.05 \mathrm{~mm}$. These findings are consistent with viewing the bridge as a (curvilinear) capillary slot, which is horizontal for the presented case.

The most significant feature of the above results is that, in all cases, the capillary force linking the two spheres across the capillary bridge eventually tends toward zero. There was not a single case where a spike in the force value was observed prior to rupture. A dispersion of data is relatively modest, except for the very end of the process when the liquid volume is minute, in particular for the very small separations.

For an evaporating capillary bridge, no theoretical predictions are available currently to compare with the obtained results (see Discussion below). However, we have performed extension tests of capillary bridges at constant volume and measured the capillary forces as they vary with the increasing separation. Such experiments have been performed numerous times before and theoretical predictions developed under different sets of hypotheses are available [15-17] for validation of the results.

The capillary force evolution during extension (Fig.4c) is visibly different from the case where the driving variable is evaporation. In the extension case, the dominant part is characterized by a continuous decrease in the force (with a 
Fig. 5 Evolution of a capillary bridge during evaporation for two spheres with an initial volume of water of $4 \mu 1$ and a separation distance of $0.7 \mathrm{~mm}$ : $(\mathbf{a}, \mathbf{c})$ thinning water bridge versus total volume of water, (b, d) evolution of the $4 \mu 1$ capillary bridge during an imposed increase in separation (extension) of two wetted glass spheres (separation in $\mathrm{mm}$ )
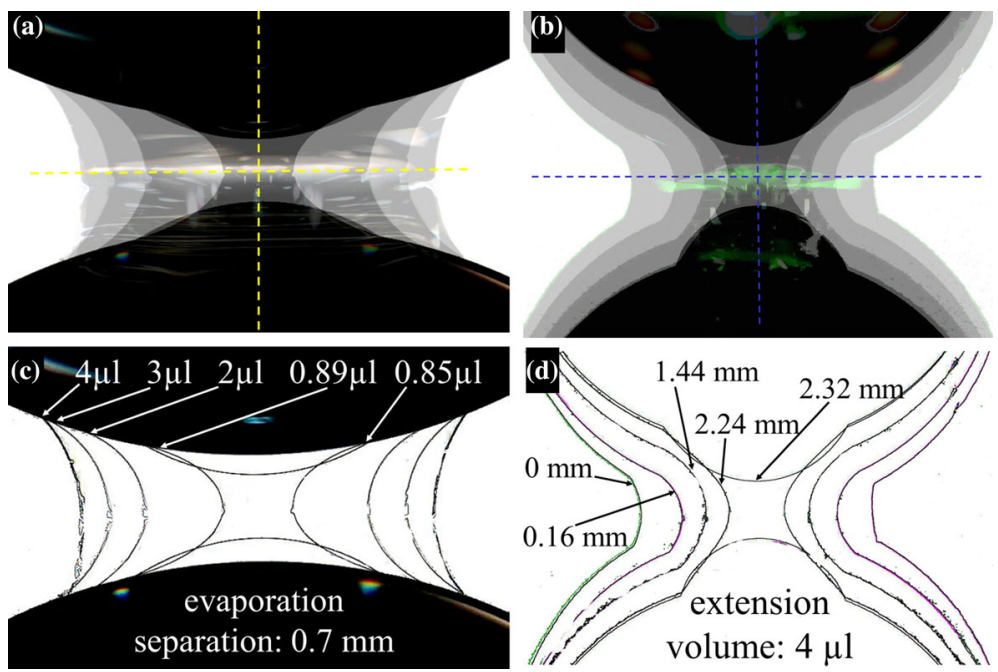

brief initial exception, see below) to terminate at very small values. This result is in agreement with the intuitive understanding of the bridge as a narrow (curvilinear) slot, the width of which is being continuously enlarged. The terminal values are coincident with a geometrical rupture and uniformly occur at approximately $10 \%$ of the initial force for all volumes. The terminal separations differ in a characteristic way: the larger the liquid volume, the larger the separation at rupture. The largest separation at rupture is approximately two times larger than the smallest separation for the volume 20 times larger. The terminal separations at rupture during extension are very near to those predicted using formulae developed earlier by Willet et al. [15] and Lian et al. [27] as shown by Mielniczuk et al. [46]. As opposed to the evaporation process, the decline of the capillary force during extension is initially high and decelerates as the process progresses, making the force-separation curve generally concave rather than convex (as during evaporation).

\subsection{Evolution of the liquid bridge shape}

To put the capillary force evolution in a wider context and to correlate it with changes of the bridge geometric parameters, a sequence of photographic images was taken every minute during each experiment. The geometrical parameters of the shape of the liquid bridge shape were processed based on the photos.

As an example of the evolution during evaporation, the shape of the liquid bridge between two vertically arranged spheres for a particle separation of $0.7 \mathrm{~mm}$ with an initial liquid volume of $4 \mu \mathrm{l}$ is shown in Fig. 5a. After a period of relatively slow evolution, which lasts for $46 \mathrm{~min}$, the total bridge volume is approximately $0.89 \mu 1$, which is less than $1 / 4$ of the original volume. At this point, changes occur rapidly, with the gorge radius decreasing within milliseconds, transforming the bridge into a central water wire and eventually ending with bridge rupture and splitting the remaining water into two separate water bodies. Figure 5a displays the images before (at $0.89 \mu \mathrm{l})$ and after rupture $(0.85 \mu \mathrm{l})$. A more detailed analysis of rupture is presented in [46].

In analogous way as above the images from the mechanical extension tests (Fig. 5b) were recorded, with the objective of wide-range comparisons with the evaporation tests. The sequence of the forms of the liquid bridge during the extension (Fig. 5b) is apparently quite similar to the sequence of forms during evaporation (Fig. 5a), but there are a few distinct differences. It is apparent that the two processes occur under different conditions: extension occurs at a constant volume and variable separation, while evaporation occurs at a constant separation and variable volume. Obviously, the overall aspect ratio of the water body evolves in a substantially different way: in evaporation, the main effect is the gorge radius shrinking, in extension the height growth is the main factor. Moreover, Fig. 5a-d demonstrates that, while the meridians of the subsequent profiles in the case of extension are roughly parallel, the meridians during evaporation with constant separation are much less self-similar; additionally, the profile becomes parabolic.

The site of liquid/solid/air contact is of crucial interest as we draw an analogy to sessile droplet evaporation. It is discussed in the following section.

\subsection{Contact angle and contact diameter}

At the first instant prior to the onset of evaporation, the capillary bridge is in thermodynamic equilibrium, which is described by Laplace-Young's law. The law states that the Laplace pressure, which is the pressure difference between the external gas and the internal fluid, depends only on, and is proportional to the mean principal curvature of the gas-liquid interface and surface tension [26]. The mechanical equilibrium implies that the mean curvature is constant for the entire 
free surface. It also implies that the gas/liquid interface surface intersects the solid surface at a constant equilibrium contact angle, $C A$ (or Young's angle, $\theta$ ). The equilibrium CA can be calculated from the values of the surface tension coefficients: $\cos \theta=\left(\gamma_{S}-\gamma_{S L}\right) / \gamma_{L}$, where the $\gamma^{\prime} s$ are the coefficients of the surface tension, with $i=S, V$ and $L$ standing for solid, vapor and liquid, respectively [26]. The nominal equilibrium contact angle for clean glass and water, is listed as zero [47-49].

However, as mentioned above, the equilibrium contact angle is regarded as an unmeasurable physical value [24,33]. The actual measurable contact angle (or a "diversity" of them) depends on the definition, the length-scale at which the three phase contact is considered, and the measurement technique $[31,50]$. The "apparent" $C A$ measured at some distance from the "triple" line, is commonly considered as the actual $C A$ [31]. In this paper contact angles are defined by points $0.5 \mu \mathrm{m}$ away from the triple line [51]. It is known to range between the advancing $C A$ value and the receding $C A$ value, and their difference is called $C A$ hysteresis (see e.g. [52]). At a micro-scale the $C A$ is affected by the molecular and microscopic structure of the substrate and possible chemical impurities $[31,50]$. As no such details are measured in our experiments, these $C A s$ are beyond our interest.

Due to the adopted procedure of constructing the liquid bridge by injecting water onto the top sphere and letting it drip by gravity to the bottom sphere, the initial top $C A s$ are at their receding values (between $15^{\circ}$ and $30^{\circ}$ ). They are much lower than their bottom counterparts, which are at their advancing values (between $35^{\circ}$ and $50^{\circ}$ ).

Another measurable geometrical characteristics of the capillary bridge is the contact diameter (CD). It is defined as an average value of the measurable radius, $r_{c o n}$, at the four visible triple contact point, multiplied by two, which amounts to assuming the contact line as a circle, Fig. 2a. It is alternatively expressed through half-filling angle, $\delta$, as $2 R \sin \delta=2 r_{\text {con }}$.

For the evaporation bridges, the evolution of the contact angles and contact diameter is substantial. It is dominated by two effects, known in the literature of sessile drop evaporation as pinning and depinning (see e.g. [19-21,34,53]. Pinning consists in contact diameter being arrested, while the evolution of contact angle proceeds. Orejon et al. [35] and Bormashenko [31] have introduced the concept of pinning force arising at the contact (or triple line) when its motion is stopped by a substrate inhomogeneity. Depinning occurs when a pinned diameter start moving at a point, while often the evolution of contact angle is stopped. Hence, a depinning force is a value of the pinning force at the moment of deactivation of the triple line. This complementarity condition for $C A$ and $C D$, is very characteristic for the behavior of the evaporating sessile drops. However, mixed modes have also been observed, and notably a slip and stick mode, of intermit- tent activation and deactivation of both motions (Orejon et al. [35]). During evaporation of the liquid bridges a very similar patterns are observed. First, the initial bottom sphere advancing $C A s$ resulting from the above described liquid insertion procedure, quite quickly convert into much smaller receding angles, despite the fact that contact diameter is pinned. Also, eventhough the $C A s$ at the top and bottom spheres are initially differentiated, depinning at the top and the bottom spheres occurs practically simultaneously. Still, the values of the depinning angle are distinctly higher for the bottom than for the top sphere, with differences on the order of 10 degrees. Because the bottom and top spheres are practically identical, the condition for the depinning of $C A$ does not seem to be linked to the sphere roughness or to precipitated impurities, but rather to their history of advancing or receding. The contact diameters at the top and bottom spheres evolve almost identically. The images in Fig. 6a and b show the mean apparent contact angle and the mean diameter at different separations. It can be seen in the figure that, for narrow separations $(0.4$ and $0.7 \mathrm{~mm})$, i.e. flat bridges, there is a period of initial $C D$ pinning, which lasts until $25-30 \%$ of the original liquid volume has been evaporated. At that point, the mean contact angle, which initially decreases quite quickly from the 30 $35^{\circ}$ range, stabilizes at a value of $13-17^{\circ}$, while the mean contact diameter undergoes depinning and starts to decrease until the bridge ruptures. For very flat and wide bridges, with separations $\leq 0.1 \mathrm{~mm}$, there is an initial, but not very consistent $C D$ pinning, with a slightly decreasing $C A$, but followed by a gradual shrinking of $C D$, while $C A$ consistently returns to the original values and above. For wider separations, either $C D$ is pinned and $C A$ is decreasing all the time $(1.3 \mathrm{~mm})$, or there is a mixed mode where $C A$ and $C D$ are both pinned (2 mm).

Interestingly, the pattern is distinctly different in the case of liquid bridge extension (Fig. 6c). Both contact angles and the contact diameter decrease initially up to separations that range from 0.5 to $1.25 \mathrm{~mm}$ (i.e. 0.625 and 0.105 of relative separations) and then stabilize at approximately the same time. However, towards the end of the process, $C A$ begins to increase slightly. Larger water volumes correspond to terminal $C A s$ and $C D s$ that are higher in value.

Surprisingly, despite a large amount of literature on liquid bridge extension, not much data are provided regarding $C A$ and $C D$. Interestingly, Willett et al. [42] found large differences in the capillary force in a bridge between two spheres when extending or compressing the bridge and attributed them to a receding or advancing contact angle.

One can summarize the above patterns as follows: during evaporation, the pinning of the contact diameter occurs at the beginning of the process (or throughout the whole process for wide separations) associated with a decreasing $C A$. After the depinning of $C D$, contact angle is pinned (or reverses) and the $C D$ decreases to zero. The situation is different dur- 

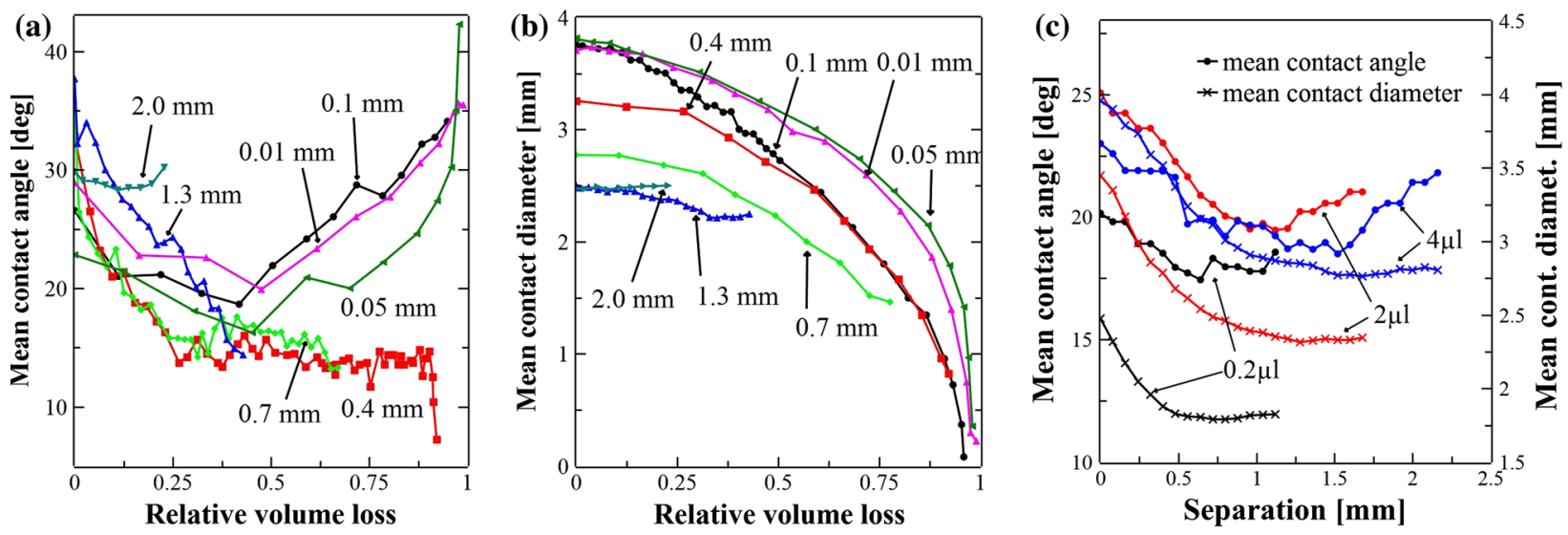

Fig. 6 Mean contact angle and mean contact diameter evolution $(\mathbf{a}, \mathbf{b})$ during evaporation tests with $4 \mu 1$ of water initially, (c) extension tests for various liquid volumes

ing extension: the first part of the process proceeds with a dynamic triple contact until the contact diameter is eventually pinned at end of the process. Interestingly, the angle behaves the same way, decreasing initially and pinned toward the end.

There is an abundance of theories for the evaporation of sessile drops about the causes and mechanisms involved in the pinning and depinning of the triple line, including solid surface roughness [54,55], the effect of triple line extension [56], dissolution of atmospheric impurities [52], hydrophobic or rough local spots [57], internal liquid flows, and nonuniform evaporation flux [58]. Because such studies require the detailed knowledge of the micro-scale surface roughness characteristics, they are beyond the scope of this paper.

\section{Discussion}

In what follows we attempt to interpret the findings about the capillary bridge evaporation in view of the known previous results concerning sessile drop drying and of the results of the extension tests. Let us reiterate that, to our knowledge, no broad-scope test results of the evaporation of liquid bridges are available, so there are not much of data for a direct comparison.

The comparison with the extension process of the capillary bridge shows analogies and differences in the bridge evolution. The process during evaporation is controlled by a decreasing volume of the liquid bridge (at constant separation). During extension the liquid volume varies imperceptibly, while separation increases.

To better visualize and understand the capillary force evolution, a combined 3D graph of the capillary force change is presented in Fig. 7a with both evaporation (constant separation) and extension (constant liquid volume). In general, the capillary force decreases in both processes, but the evaporation force $F_{C A P}$ versus the relative lost evaporated liquid volume is a convex function (slow changes at the beginning and function fast changes at the end), while the extension force $F_{C A P}$ versus separation is a concave (fast changes at the beginning and slow at the end).

A fundamental question arises, which is whether or not the capillary force between two glass beads is a state variable that depends only on the current liquid bridge volume and current separation. In the case of the affirmative answer, that would imply that both families of curves shown in Fig. 7a would form a unique surface. This is clearly not the case, or at least, there are notable discrepancies in certain ranges of the variables. The capillary force differ visibly, especially in the range of small volumes and small separations, where the values for $F_{C A P}$ are up to two times lower for the evaporation processes than during extension. To render the numerical differences more visible, hypothetical curves of the capillary force at specific separations obtained for the indicated current liquid volume experiments are constructed and projected on a capillary-force-versus-volume plane in Fig. 7b. These curves are to be compared to the actual experimental curves for evaporation. Similarly, in Fig. 7c, the capillary force vs. indicated separations are constructed for specific current volumes and projected on a capillary-force-versus-separation plane.

There appears to be two types of discrepancies in two different ranges. First, a substantial difference arises from the fact that the surfaces $F_{C A P}(V, D)$ that hypothetically span the constant separation (evaporation) curves and those that span the constant volume (extension) curves do not exist for certain liquid volume values. These values where the hypothetical surface for evaporation does not exist include a relatively vast range of the total liquid volumes; as an example, for the small volumes (e.g., less than $2 \mu 1$ at $D=1.3 \mathrm{~mm}$ and less than $1 \mu \mathrm{l}$, at $D=0.7 \mathrm{~mm}$ ) that are marked on the "floor" of the graph in Fig. 7a, the hypothetical force surface for evaporation does not exist, but it does appear to exist for 

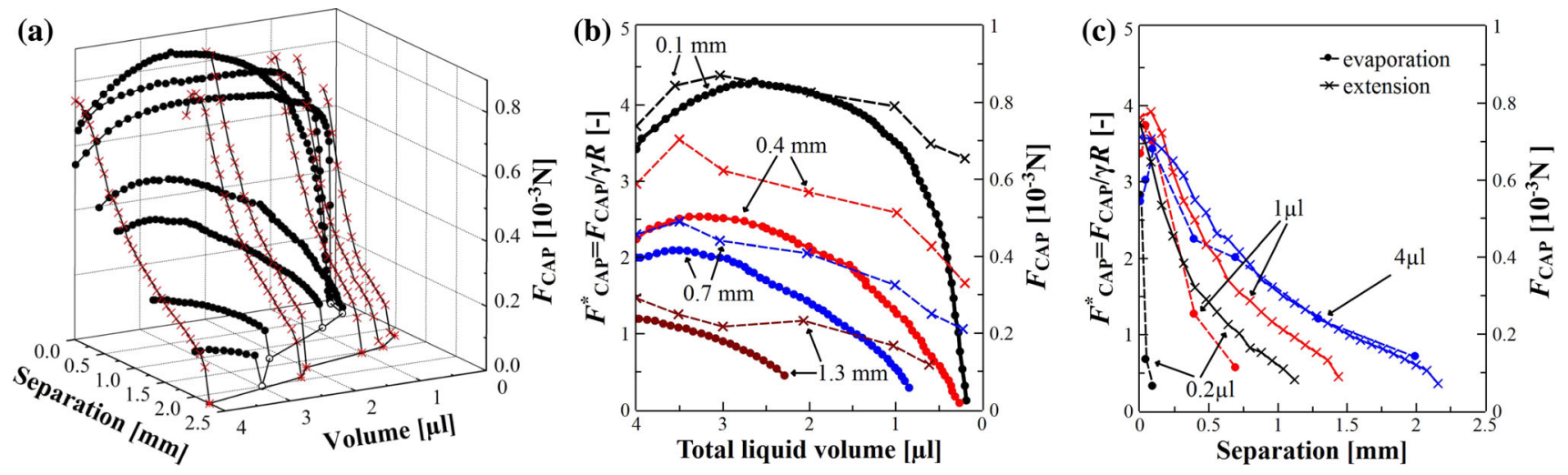

Fig. 7 Evolution of the total capillary force at the gorge of a liquid bridge. (a) A 3-D representation of the capillary force for the constant separation evaporation tests (dots) and the constant volume extension tests (crosses); (b) The capillary force during the evaporation tests for selected constant separations (dots) versus the values of the force at the corresponding separations (crosses) in the extension tests at a specific liquid volume of the bridge (connected by a hypothetical constant sep- aration curve, shown as dashed line). Note the discrepancy between the two types of constant separation lines for the two tests at smaller liquid volumes; (c) The capillary force during extension tests for selected constant volumes (crosses) versus the values of the force (small dots) at the corresponding volumes in the evaporation tests at specific separations (connected by a hypothetical constant volume curve, shown as dashed line) extension. A clear cause of these discrepancies is the occurrence of rupture of the tall and slender bridges relatively early during evaporation at wider separations. At the same time, the bridges remain stable for the same volumes during extension.

Additionally, even prior to rupture, in the ranges where the force exists for both the constant volume and constant separation processes, there are differences in the capillary forces that may be as large as $80 \%$ of the maximum value for a constant separation process $(0.1 \mathrm{~mm})$ or $92 \%$ for a constant volume process $(0.2 \mu \mathrm{l})$. The forces for the constant volume conditions (extension) are consistently higher than the forces in the constant separation process (evaporation).

The above findings support the conclusion that in general, the evolving capillary forces in a liquid bridge are not uniquely defined by the current liquid volume $V$ and the separation $D$. Thus, it appears that the capillary force during evaporation is not a state function of the liquid volume and separation. Nevertheless, the relationship between $F_{C A P}, V$ and $D$ for constant separations and for constant volume seems to be closer at larger volume values. The discrepancy for larger separations seems to be related to the bridge rupture. However, for smaller separations, where both the evaporation and extension functions exist in the entire domain, additional mechanisms and/or variables must be considered to explain the discrepancies between the two functions (see below).

The capillary force data may be compared to the calculated values obtained by Mielniczuk et al. [37], which were based on the measurements of the gorge radius of curvature and on the averaged external radius. From these radii it is possible to calculate the value of the Laplace pressure, while from the external radius the surface tension force at the gorge and hence the corresponding resultant total capillary force.
Figure 8a, $\mathrm{b}$ show the evolution of these forces for the evaporation and extension tests and directly compares them to the measured forces in Fig. 7. It should be noted that the calculation of the forces is based on the assumption that the process is not far from equilibrium.

The comparison with the directly measured force is quite good for the evaporation process at larger separations; however, for smaller separations (less than $0.4 \mathrm{~mm}$ ), there is a sizable discrepancy, almost $50 \%$ for $D=0.01 \mathrm{~mm}$, where even the qualitative trend is not maintained. For smaller separations and a specific volume of liquid, the middle phase of the process is dominated by suction while the latter phase of the process is dominated by the surface tension (see [37] for details).

One noticeable anomaly in the calculated forces is that the force magnitude at the extremely small separation of $0.01 \mathrm{~mm}$ compared to that of $0.05 \mathrm{~mm}$, which does not follow the trend for larger separations. The tests were repeated several times and the results appeared to persist, suggesting that either our measurements at these small separations are biased or there are interfering short-distance phenomena that we do not consider. However, based on Molecular Dynamics simulations of the capillary bridges, even the smallest separations are still within the range of validity of Kelvin theory [45]. In general, at least for larger and moderate separations, the comparison provides an indirect confirmation that the adopted experimental techniques are sufficiently accurate for the present purpose.

The resultant of the capillary force during extension (Fig. 8b) is in good agreement with the forces calculated from the imaged radii. The largest discrepancy, which is still less than $15 \%$, occurs at the beginning of the test with the largest volume of liquid. 


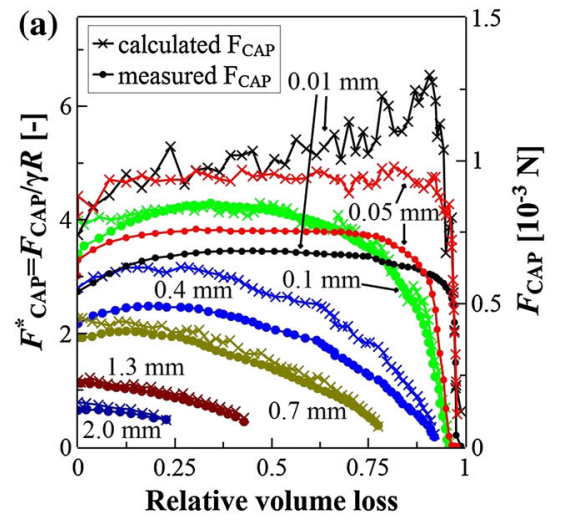

Fig. 8 Total resultant of the capillary force between two spheres: a comparison between the measured force and the force calculated from the curvature radii by Mielniczuk et al. [37]: (a) during evaporation; (b) during extension; (c) a comparison for the extension test a theoret-

For the constant volume process, a number of "theoretical" solutions for the capillary force have been derived by minimizing the surface energy. A closed-form solution by Lian et al. [27], as approximated in [59] and [38,60], has been compared to the measured data in [37] and shown in Fig. 8c.

The closed-form solution clearly leads to a systematic overestimation of the force in the initial instant and at initial separations. This discrepancy has been reported for numerous experiments (see, e.g., $[15,27,38,59,60]$ ) and is commonly attributed to the variability of the contact angle and pinning of the triple line [60], which are not part of the classical description of the surface energy.

For the evaporation of sessile drops, the continuous loss of liquid mass due to evaporation is seen as a cause of the droplet profile deviating from the initial equilibrium profile. In turn, Bourgès-Monnier and Shanahan [21] found that the Young's force (surface tension) deviates from the equilibrium value, which generates an additional anchoring force at the triple line that controls the actual equilibrium of the contact.

The most visible aspect of the liquid bridge evolution, which does not appear explicitly in the Laplace-Young law, is indeed the dynamics of the contact line. This dynamics is characterized by three variables: the contact force, the contact diameter and the contact angle. The first is not measurable with our setup. The evolution of the contact diameter and the contact angle are shown in Fig. 6 and were discussed in a previous section. In Fig. 9a, b we present the relationships between the capillary force and the mean contact angle and between the capillary force and the mean contact diameter for the bridge evaporation process, respectively. Figure 10a,b present the same relationships for the bridge extension.

It is apparent from Figs. 9 and 10 that the presented curves are quite different for evaporation and for extension. During the first phase of evaporation, which is associated with the pinning of $C D$ and a consistent decrease of $C A$, the capillary force either barely changes, or increases slightly for medium and narrow separations, or decreases slightly for wide separations. Therefore, the force is practically independent of the apparent contact angle, Fig. 9a. Once depinning has occurred (marked with a vertical arrow, Fig. 9a), the dependence of the capillary force on the apparent contact angle changes dramatically. In general, there is an interval where $C A$ decreases slightly, after which the force drastically decreases to zero (or to rupture for 1.3 and $2 \mathrm{~mm}$ ), which occurs at $C A$ value that is almost constant. This trend is visible to different extents for different widths of separation.

For the contact diameter (Fig. 9b) a small increase in the force occurs for evaporation at a near-constant (pinning) or slightly decreasing contact diameter, after which there is a gradual decrease of the capillary force with the diameter decreasing, with nearly the same rate for all separations. Hence, the capillary force does not depend on the contact angle during evaporation, but it depends on the threshold contact angle at depinning.

The depinning occurs at different capillary force values for different separations. However, the values of the contact angle at depinning are close to one another: approximately $14-20^{\circ}$ for all the separation of $0.01,0.1,0.4,0.7$ and $1.3 \mathrm{~mm}$ but it is $27^{\circ}$ for $2 \mathrm{~mm}$. There is a correlation of the depinning with the advancement of evaporation (see Fig. 6a), depinning consistently occurs earlier and at a smaller relative evaporated volume for the bridge width increasing (i.e., as the bridge height or slenderness is bigger).

No criterion hypothesis for depinning is advanced at this point. In the sessile droplet evaporation literature the predominant view is that there is a micro-scale energy barrier that needs to be overcome to initiate the triple line motion, see e.g., [61,62]. Most commonly, the resulting criteria involve surface roughness and liquid impurity or precipitates. Our 
Fig. 9 Capillary force during evaporation (a) versus mean apparent contact angle, and (b) versus mean contact diameter. The arrows show depinning points
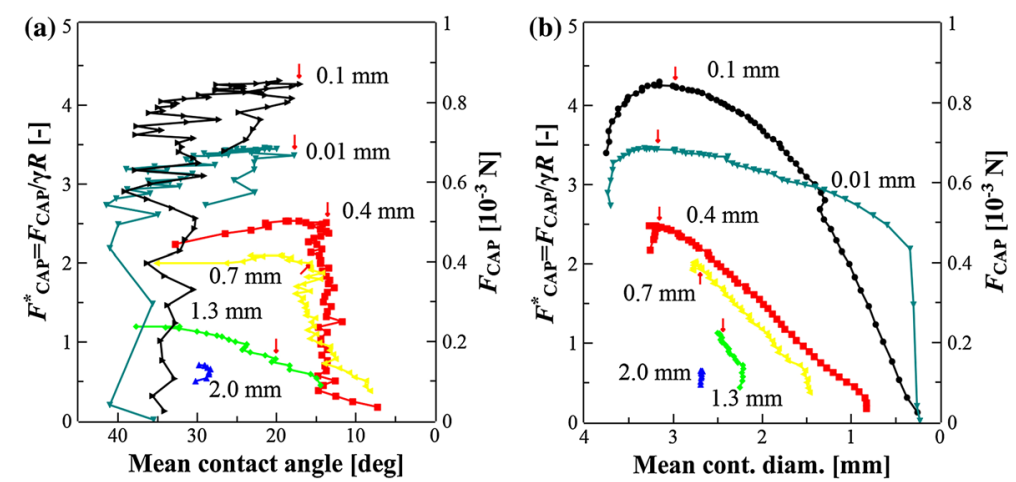

Fig. 10 Capillary force during extension (a) versus mean contact angle, and (b) versus mean contact diameter
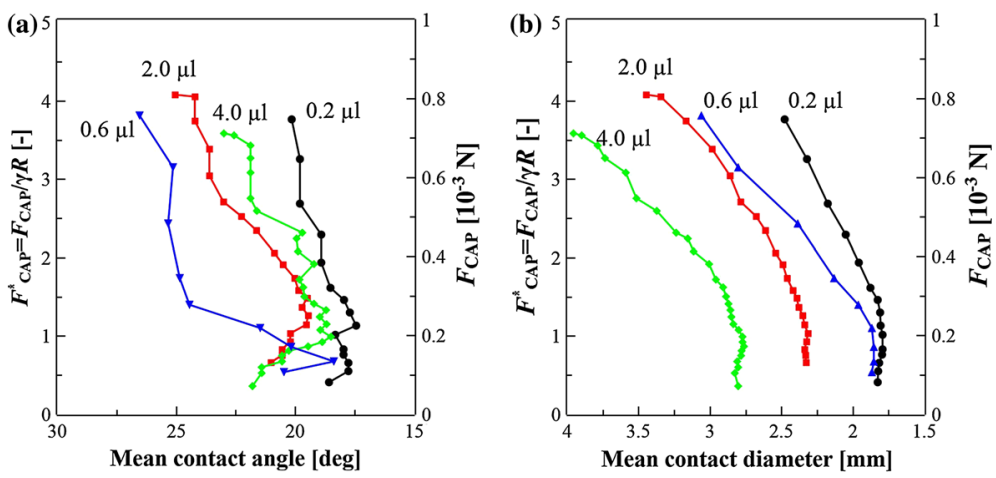

observation that depinning occurs nearly simultaneously at the top and the bottom sphere contact, but consistently at a much lower contact angle at the top sphere than at the bottom contact, the sphere roughness being equal, suggests that history of the contact (initially receding, or initially advancing) may play a role.

During extension, there is a mixed mode behavior, which means that $C A$ and $C D$ vary simultaneously; both variables either decrease or remain constant. The capillary force decreases with a constant rate for most of the process. Some $C D$ pinning is seen at the end of extension and seems to be simultaneous with the freezing of the contact angle.

Three of the important findings, the different aspect ratio of the water body, different kinematics of the triple contact line during evaporation and extension, and most importantly, the correlation between the capillary force evolution and depinning, require the above-mentioned variables that are below the macroscopic level to be considered if a realistic model of the evaporation of capillary bridges were to be pursued.

\section{Conclusions}

Apart from a slight initial increase at narrow separations, the capillary force between two spheres consistently decreases to zero upon evaporation, unless the capillary bridge ruptures mid-way, which happens mainly for large separations (i.e., tall and thin bridges). At these separations, the rupture of the bridge may occur at an early stage of the water mass loss, with an up to $75 \%$ of the capillary water still in the system. For all other separations, the capillary force decreases to zero as the water evaporates completely. For very narrow separations, the bridge ends up evolving in an unstable manner.

In none of the examined cases did the capillary force increase significantly. The fact that the interparticle capillary force decreases to zero during drying of the bridges is of importance to the mechanics of unsaturated granular media, where a substantial growth of "apparent" cohesion during the terminal stage of desaturation is commonly attributed to an increase of intergranular forces and suction. The latter statement is usually substantiated by so-called characteristic curves that establish a one-to-one relationship between the macro-scale variables of suction and degree of saturation in suction-driven dehydration experiments $[63,64]$. Two-grain bridges are often the last stage of drying of larger capillary bridges that span multiple grains. Nevertheless, the intergranular forces were seen to decrease to zero upon drying in multi-grain bridges $[9,65]$.

It appears that the capillary force in a liquid bridge during evaporation and during extension cannot be represented with the same functional dependence on the total liquid volume and the grain separation. Thus, the capillary force is not a function of state during evaporation. First, the capillary force may disappear because of bridge rupture in a significant range of the variables, especially for larger separations and for small water volumes. Second, there are significant differ- 
ences in the capillary force value over the rest of the range. One likely origin for the differences is the evolution of the triple contact line, and in particular, its tendency towards initial pinning during evaporation, associated with the contact angle decrease at a roughly constant capillary force, followed by a depinning at a contact angle that becomes nearly constant, while the force precipitously decreases to zero, roughly proportional to the contact diameter change.

Hence, the onset of the decrease in the capillary force is closely correlated with two crucial characteristics of the bridge evolution: depinning, i.e. the onset of the decrease in the contact diameter, and the arrest of the decrease of the contact angle. This suggests that the evolution, or the history, of the triple line is crucial for the capillary force evolution during the evaporation of the liquid bridge and that it cannot be ignored in the analysis and modeling of the liquid bridge evaporation. However, the determination of the depinning criteria likely requires a micro-scale analysis, as for drying of a sessile drop. In addition, the results suggest that a depinning criterion should include the history (during bridge formation) of the apparent contact line advancement or recession.

All the presented results concern granular systems that are characterized by constant separations and contain water only within the inter-particle bridges; hence, their water content is low. Thus, the above considerations apply only to the terminal drying phase of granular materials.

Acknowledgments The authors acknowledge the partial support of US NSF Grant \#0700294 of CMMI Division, Geomechanics and Geomaterials Program (T. Hueckel) and the support of CNRS, IRSN (MIST laboratory) and the Languedoc-Roussillon region (B. Mielniczuk, M. S. El Youssoufi, T. Hueckel).

\section{References}

1. Peron, H., Delenne, J.Y., Laloui, L., El Youssoufi, M.S.: Discrete element modelling of drying shrinkage and cracking of soils. Comput. Geotech. 36, 61-69 (2009)

2. Hu, L.B., Peron, H., Hueckel, T., Laloui, L.: Desiccation shrinkage of non-clayey soils: multi-physics mechanisms and a microstructural model. Int. J. Numer. Anal. Methods Geomech. (2012). doi: $10.1002 /$ nag. 2108

3. Hu, L.B., Peron, H., Hueckel, T., Laloui, L.: Desiccation shrinkage of non-clayey soils: a numerical study. Int. J. Numer. Anal. Methods Geomech. (2012). doi:10.1002/nag.2107

4. Hu, L.B., Peron, H., Laloui, L., Hueckel, T.: A multi-scale multiphysics model of soil drying. ASCE Geotechnical Special Publication 211: Geo-Frontiers 2011, Advances in Geotechnical Engineering, Dallas, TX, USA, pp. 4349-4358 (2011)

5. Hu, L.B., Peron, H., Hueckel, T., Laloui, L.: Drying shrinkage of deformable porous media: mechanisms induced by the fluid removal. In: Olson, H.W. (ed.) ASCE Geotechnical Special Publication 157: Computer Applications in Geotechnical Engineering, Geo-Denver 2007 (CD-ROM) (2007)
6. Kowalski, S.J., Mielniczuk, B.: Analysis of effectiveness and stress development during convective and microwave drying. Dry Technol. 26, 64-77 (2008)

7. El Youssoufi, M.S., Delenne, J.Y., Radjai, F.: Self-stresses and crack formation by particle swelling in cohesive granular media. Phys. Rev. E 71, 051307 (2005)

8. Urso, M.E.D., Lawrence, ChJ, Adams, M.J.: A two-dimensional study of the rupture of funicular liquid bridges. Chem. Eng. Sci. 57, 677-692 (2002)

9. Hueckel, T., Mielniczuk, B., El Youssoufi, M. S.: Micro-scale study of rupture in desiccating granular media, Proceedings of Geo-Congress 2013, Geotechnical Special Publication GSP 231, pp. 808-817, ASCE (2013)

10. Harris, C.C., Morrow, N.R.: Pendular moisture in packings of equal spheres. Nature 203, 706-708 (1964)

11. Gao, C.: Theory of menisci and its applications. Appl. Phys. Lett. 71, 1801-1804 (1997)

12. Maeda, N., Israelachvili, J.N., Kohonen, M.M.: Evaporation and instabilities of microscopic capillary bridges. Proc. Natl. Acad. Sci. USA 100(3), 803-808 (2003)

13. Cutts, R.E., Burns, S.E.: Evolution of surface area-to-volume ratio for a water meniscus evaporating between contacting silica spheres. J. Colloid Interface Sci. 343, 298-300 (2010)

14. Zhou, Z., Li, Q., Zhao, X.S.: Evolution of interparticle capillary forces during drying of colloidal crystals. Langmuir 22, 3692-3697 (2006)

15. Willett, ChD, Adams, M.J., Johnson, S.A., Seville, J.P.K.: Capillary bridges between two spherical bodies. Langmuir 16, 9396-9405 (2000)

16. Rabinovich, Y.I., Esayanur, M.S., Moudgil, B.M.: Capillary forces between two spheres with a fixed volume liquid bridge: theory and experiment. Langmuir 21, 10992-10997 (2005)

17. Butt, H.-J., Kappl, M.: Normal capillary forces. Adv. Colloid Interface Sci. 146, 48-60 (2009)

18. Gras, J.-P., Delenne, J.-Y., El Youssoufi, M.S.: Study of capillary interaction between two grains: a new experimental device with suction control. Granul. Matter 15, 49-56 (2013)

19. de Gennes, P.G.: Wetting: statics and dynamics. Rev. Mod. Phys. 57, 827-863 (1985)

20. Bourgès, C., Shanahan, M.E.R.: L'influence de l'évaporation sur l'angle de contact des gouttes d'eau. C. R. Acad. Sci. Paris 316, 311-316 (1993)

21. Bourgès-Monnier, C., Shanahan, M.E.R.: Influence of evaporation on contact angle. Langmuir 11, 2820-2829 (1995)

22. Marmur, A.: Soft contact: measurement and interpretation of contact angles. Soft Matter 2, 12-17 (2006)

23. Anantharaju, N., Panchagnula, M., Net, S.: Evaporating drops on patterned surfaces: transition from pinned to moving triple line. J. Colloid Interface Sci. 337, 176-182 (2009)

24. Bormashenko, E.: Wetting of real solid surfaces: new glance on well- known problems. Colloid Polym. Sci. 291, 339-342 (2013). doi:10.1007/s00396-012-2778-8

25. Adamson, A.W.: Physical Chemistry of Surfaces, 3rd edn. Wiley, New York and London (1976)

26. Landau, L.D., Lifshitz, E.M.: Course of Theoretical Physics, Fluid Mechanics, vol. 6. Pergamon Press, Oxford (1959)

27. Lian, G., Thornton, C., Adams, M.J.: A theoretical study of the liquid bridge forces between two rigid spherical bodies. J. Colloid Interface Sci. 161, 138-147 (1993)

28. Princen, H.M.: Comments on "The effect of capillary liquid on the force of adhesion between spherical solid particles". J. Colloid Interface Sci. 26, 249-253 (1968)

29. Erbil, H.Y.: Evaporation of pure liquid sessile and spherical suspended drops: a review. Adv. Colloid Interface Sci. 170, 67-86 (2012) 
30. Marmur, A.: Wetting on real surfaces. IS\&Ts NIP 15: 1999 International Conference on Digital Printing Technologies, pp. 22-25 (1999)

31. Bormashenko, E.: Progress in understanding wetting transitions on rough surfaces. Adv. Colloid Interface Sci. (2014) doi:10.1016/j. cis.2014.02.009

32. Deegan, R.D., Bakajin, O., Dupont, T.F., Huber, G., Nagel, S.R., Witten, T.A.: Capillary flow as the cause of ring stains from dried liquid drops. Nature 389, 827-829 (1997)

33. Israelachvili, J.N.: Intermolecular and Surface Forces, 3rd edn. Academic Press, USA (2011)

34. Bormashenko, E., Musin, E.A., Whyman, G., Zinigrad, M.: Wetting transitions and depinning of the triple line. Langmuir 28, 34603464 (2012)

35. Orejon, D., Sefiane, K., Shanahan, M.E.R.: Stick-slip of evaporating droplets: substrate hydrophobicity and nanoparticle concentration. Langmuir 27, 12834-12843 (2011). doi:10.1021/ la2026736

36. Kröger, J., Rath, H.J.: Velocity and elongation rate distributions in stretched polymeric and Newtonian liquid bridges. J. Non-Newton. Fluid Mech. 57, 137-153 (1995)

37. Mielniczuk, B., Hueckel, T., El Youssoufi, M.S.: Evolution of Laplace pressure and surface tension for evaporating two-grain water bridge. Submitted

38. Soulié, F., Cherblanc, F., El Youssoufi, M.S., Saix, C.: Influence of liquid bridges on the mechanical behaviour of polydisperse granular materials. Int. J. Numer. Anal. Methods Geomech. 30, 213-228 (2006)

39. Das, S.: Effect of impurities in the description of surface nanobubbles: role of nonidealities in the surface layer. Phys. Rev. E 83, 066315 (2011)

40. Bircumshaw, L.L.: The surface tension of mixtures of alcohol and water at $25^{\circ}$. Chem. Soc. Trans. 121, 887 (1922)

41. Pepin, X., Rossetti, D., Iveson, S.M., Simons, S.J.R.: Modeling the evolution and rupture of pendular liquid bridges in the presence of large wetting hysteresis. J. Colloid Interface Sci. 232, 289-297 (2000). doi:10.1006/jcis.2000.7182

42. Willett, C.D., Adams, M.J., Johnson, S.A., Seville, J.P.K.: Effects of wetting hysteresis on pendular liquid bridges between rigid spheres. Powder Technol. 130, 63-69 (2003)

43. Megias-Alguacil, D., Gauckler, L.J.: Capillary forces between two solid spheres linked by a concave liquid bridge : regions of existence and forces mapping. AiChE J. 55, 1103-1109 (2009)

44. Adams, M.J., Johnson, S.A., Seville, J.P.K., Willett, C.D.: Mapping the influence of gravity on pendular liquid bridges between rigid spheres. Langmuir 18, 6180-6184 (2002)

45. Fisher, L.R., Israelashvili, J.N.: Experimental studies on the applicability of the Kelvin equation to highly curved concave menisci. J. Colloid Interface Sci. 80, 528-541 (1981)

46. Mielniczuk, B., El Youssoufi, M.S., Sabatier, L, Hueckel, T.: Rupture of a liquid bridge between two grains due to its evaporation. Acta Geophysica (2014). doi:10.2478/s11600-014-0225-6
47. Young, T.: An essay on the cohesion of fluids. Philos. Trans. 65, 1071 (1805). doi:10.1098/rstl.1805.0005.JSTOR59

48. Birch, W., Carré, A., Mittal, K.L.: Wettability techniques to monitor the cleanliness of surfaces, Chapter 13. In Kohli, R. and Mittal, K.L. (eds.) Developments in surface contamination and cleaning, William Andrew Inc (2008)

49. Lide, D.R. (ed.) CRC Handbook of Chemistry and Physics, 75th edition. CRC Press (1994)

50. Decker, E.L., Frank, B., Suo, Y., Garoff, S.: Physics of contact angle measurement. Colloids Surf. A Physicochem. Eng. Asp. 156, 177 189 (1999)

51. Wolansky, G., Marmur, A.: Apparent contact angles on rough surfaces: the Wenzel equation revisited. Colloids Surf. A Physicochem. Eng. Asp. 156, 381-388 (1999)

52. de Gennes, P.G., Brochard-Wyart, F., Quéré, D.: Gouttes, bulles, perles et ondes. Belin, Paris (2002)

53. Pittoni, P.G., Chang, C.-C., Yu, T.-S., Lin, S.-Y.: Evaporation of water drops on polymer surfaces: pinning, depinning and dynamics of the triple line. Colloids Surf. A Physicochem. Eng. Asp. 432, 89-98 (2013)

54. Wenzel, R.N.: Resistance of solid surfaces to wetting by water. Ind. Eng. Chem. 28, 988-994 (1936)

55. Cassie, A.B.D., Baxter, S.: Wettability of porous surfaces. S. Trans. Faraday Soc. 40, 546-551 (1944)

56. Boruvka, L., Neumann, A.W.: Generalization of the classical theory of capillarity. J. Chem. Phys. 66, 5464-5476 (1977)

57. Gao, L., McCarthy, T.J.: How Wenzel and Cassie Were Wrong. Langmuir 23, 3762-3765 (2007)

58. Eral, H.B.D'.t Mannetje, J.C.M., Oh, J.M.: Contact angle hysteresis: a review of fundamentals and applications. Colloid Polym. Sci. 291, 247-260 (2013)

59. Mikami, T., Kamiya, H., Horio, M.: Numerical simulation of cohesive powder behavior in a fluidized bed. Chem. Eng. Sci. 53, 1927 1940 (1998)

60. Soulié, F. : Cohésion par capillarité et comportement mécanique de milieux granulaires. PhD thesis, Université Montpellier 2, Montpellier (2005)

61. Marmur, A.: Wetting on hydrophobic rough surfaces: to be heterogeneous or not to be? Langmuir 19, 8343-8348 (2003)

62. Patankar, N.A.: Transition between superhydrophobic states on rough surfaces. Langmuir 20, 7097-7102 (2004)

63. Rahardjo, H., Fredlund, D.G.: Experimental verification of the theory of consolidation for unsaturated soils. Can. Geotech. J. 32, 749 (1995)

64. Peron, H., Hueckel, T., Laloui, L.: An improved volume measurement for determining soil water retention curves. Geotech. Test. J. 30, 1-8 (2007)

65. Hueckel, T., Mielniczuk, B., El Youssoufi, M.S., Hu, L.B., Laloui, L.: A three-scale cracking criterion for drying soils. Acta Geophysica (2014). doi:10.2478/s11600-014-0214-9 